\title{
Lateralization in haptic processing: An apparatus for analyzing manual strategies
}

\author{
JOËL FAGOT, BERNARD ARNAUD, MONIQUE CHIAMBRETTO, and RAYMOND FAYOLLE \\ CNRS, Laboratoire de Neurosciences Fonctionnelles, Unité de Neurosciences Cognitives \\ Marseille, France
}

\begin{abstract}
We describe an apparatus for testing laterality in haptic processing in the discrimination of nonsense shapes in humans or monkeys. The system, which permits either mono- or dichhaptic discrimination, automatically provides data on the measurement of accuracy along with information on hand exploratory strategies.
\end{abstract}

Lateralization in haptic processing in humans has been studied in a variety of ways and has given rise to a vast literature (for a review, see Summers \& Lederman, 1990). Although the results are still controversial, studies have repeatedly demonstrated a left-hand advantage for discriminating meaningless shapes. This left-hand superiority might in part be due to better processing of spatial information by the right hemisphere (see, e.g., Gardner et al., 1977; Grogan, 1986). Interestingly, asymmetry in favor of the left hand in tactile discrimination has also been found in rhesus monkeys (Fagot, Dréa, \& Wallen, 1991; Hoerster \& Ettlinger, 1985).

In most studies, the stimuli are plastic or wood blocks of different shapes hidden from view that the subjects have to touch either with one hand (monohaptic exploration) or with both hands (dichhaptic exploration; see Witelson, 1974) and then recognize (see, e.g., Lenhart \& Schwartz, 1983). The dependent variable is usually the number of accurate responses for the left and for the right hand. Under the assumption that a hand preference or advantage reveals hemispheric differences in spatial abilities, another interesting approach would be to analyze comparatively exploratory procedures of each hand. For example, on the basis of the serial/parallel (Cohen, 1973) or analytic/holistic dichotomies (Nebes, 1978), one would expect that the right hand (left hemisphere) sequentially investigates the specific features of the shape, whereas the left hand (right hemisphere) explores in a more global manner. The apparatuses available to date do not address such questions.

We describe a new instrument designed to answer the critical question of laterality in hand exploratory strategies. This apparatus fulfills the following methodological demands. First, because our lab uses a comparative

\footnotetext{
We would like to thank V. Ginouvier for the preparation of Figure 1. We are also grateful to Jacques Vauclair (CNRS, Marseille, France) and Kim Wallen (Emory University, Atlanta) for their helpul comments on the first draft of this paper. Correspondence should be sent to Joell Fagot, CNRS, Laboratoire de Neurosciences Fonctionnelles, Unité de Neurosciences Cognitives, 31 chemin Joseph Aiguier, 13409 Marseille Cedex 09, France.
}

approach, we wanted a single instrument to test both humans and monkeys. Second, we wanted to be able to investigate mono- and dichhaptic exploration with the same apparatus. Third, we needed to record accuracy along with spatial and temporal information about how the left and right hands touch the shapes, in order to evaluate the consistency between these different indices of lateralization.

\section{STIMULI, APPARATUS, AND PROCEDURE}

Subjects haptically investigate one or two stimuli consisting of 1-12 square metallic touch units (Figure 1A) that are pegged side by side on the vertical rear panel of a box-shaped apparatus. The apparatus, inside which the touch units are pegged, is depicted in Figure 1B. In brief, it has three important characteristics. First, it is made of aluminum, thus concealing the stimuli from view. Second, its front side is equipped with two motors. A vertically sliding door is suspended by a small cord to the cam of each motor. A door opening provides a $7 \times 14.5 \mathrm{~cm}$ access to the tactile stimuli. Third, depending on whether the experimental procedure requires mono- or dichhaptic discrimination, the vertical rear door may be equipped with one plastic panel $(15.4 \times 16.4 \mathrm{~cm})$ in its center or with two plastic panels placed side by side $(15.4 \times 16.4 \mathrm{~cm}$; see Figure 1C), on which the touch units are installed. One hundred forty-four holes in a $12 \times 12$ matrix are drilled in each panel. Each hole is fitted with a socket for holding a touch unit. The sockets are electrically connected on the back of the panel to a system (described later) that allows the determination of the spatial location of the pegged touch units by means of the $x, y$ coordinates of the matrix. The panels are mounted on springs above microswitches. Pushing a panel backward activates four microswitches fixed on its backside (see Figure 1C).

On a typical trial, the subject sits in front of the apparatus. The experimenter arranges the stimuli on the rear panel of the apparatus as illustrated in Figure 1C. A skillful experimenter needs approximately $80 \mathrm{sec}$ to arrange a form made of 12 touch units. ${ }^{1}$ The experimenter starts the test by pressing a key on the computer's keyboard. The computer then delivers an auditory warning signal, 


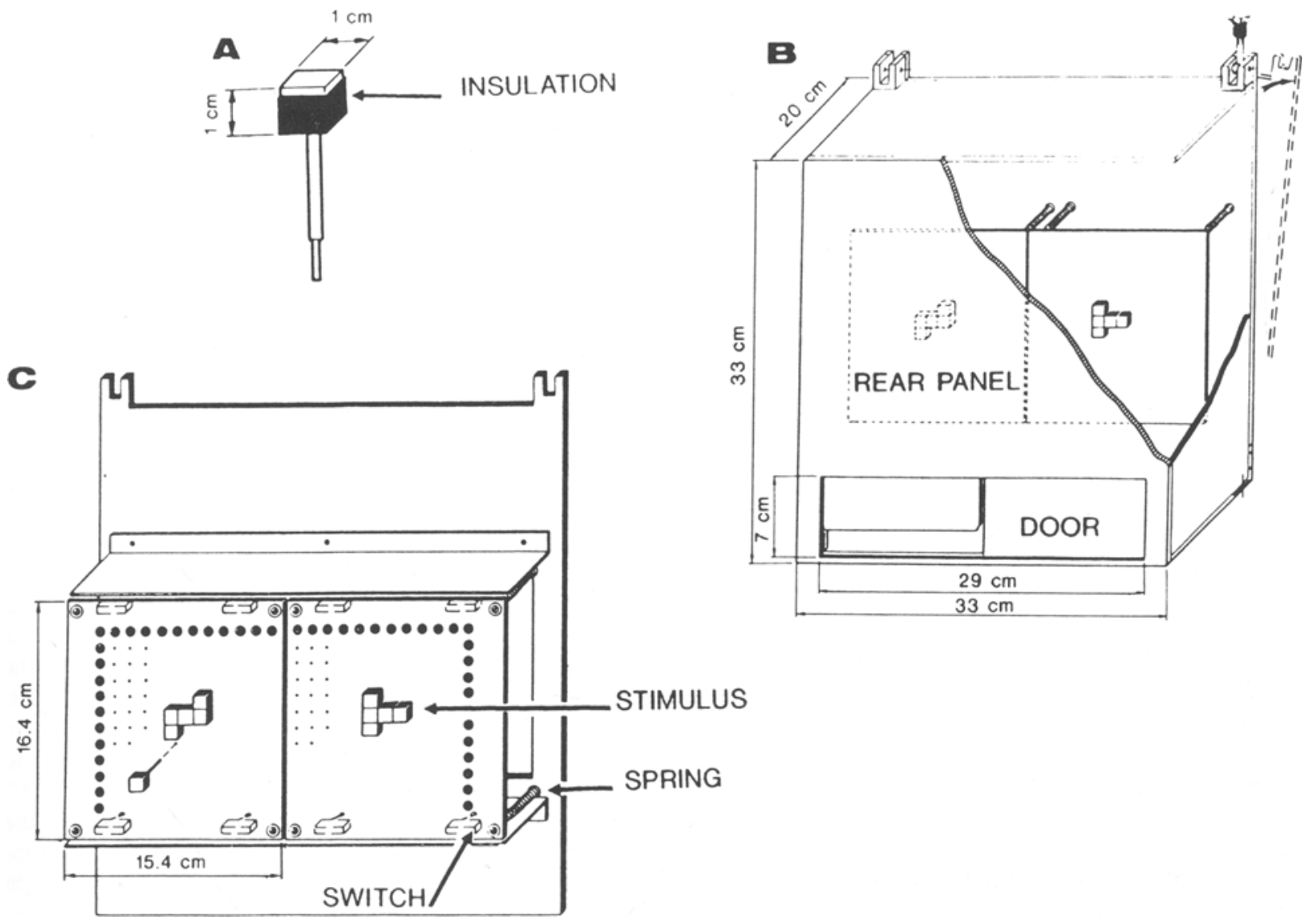

Figure 1. Three views of the apparatus and of the touch units. (A) Touch unit. (B) Front view of the apparatus. (C) Front view of the rear door of the apparatus. All measurements are in centimeters.

and a few seconds later, it triggers the opening of the left door, the right door, or both doors, depending on which key has been pressed. If the left door or the right door is opened, the subject then inserts, respectively, the left or the right hand inside the apparatus in order to touch the stimuli. If both doors are opened, the subject then inserts both hands simultaneously inside the apparatus to perform a dichhaptic exploration. The springs on which the panels are mounted are sufficiently hard to avoid accidental panel pushes during the palpation phase. Because the subject is grounded and the metallic touch units are positively polarized, a finger contact with one part of the stimulus changes the electrical state of that part. That change is recorded by the computer. The subject's response is a push on the panel containing the "correct" stimulus. The push causes the door(s) to close and thus ends the trial. If no decision is given, a warning tone is delivered at the end of the exploratory time delay, and the door(s) is (are) closed.

\section{ELECTRONIC COMPONENTS}

Figure 2 depicts the principal constituents of the system and their connections. In addition to the discriminanda de- scribed above, the apparatus requires an IBM-compatible computer equipped with a VGA monitor $(640 \times 480)$ and two digital I/O cards (DT 2817; Data Translation Inc., Marlboro, MA). The computer is connected, via these cards and a digital I/O bus, to a multifunction interface that, in turn, is linked to the apparatus. For testing monkeys, a food dispenser for Noyes pellets (Model 442; Gerbrands, Arlington, MA) and its box relay are also attached to the multifunction interface.

Shown in Figure 3 are the four separate circuits used for (1) localizing the pegs on each panel; (2) opening and closing the front doors; (3) detecting hand contacts with the touch units; and (4) detecting panel pushes. In brief, the localization of the touch units on each panel (Figure $3 \mathrm{~A}$ ) requires a sequential scanning of the rows and columns formed by the $12 \times 12$ matrix of sockets (KLB1; Lumberg GMBH, Schalksmühle, Germany). Outputs from a multiplexer (74LS5150) are driven to the computer via the digital $\mathrm{I} / \mathrm{O}$ bus. The opening and closing of the door (Figure 3B) is accomplished with a stepping motor (P430-238; Nippon Pulse Motor Co., Tokyo) activated by a stepper motor driver receiving clock signals from a timer (UA555) either (1) through a microswitch (B62-20Y; Bonnella Switches, Chettenham, U.K.) fixed on the cam of 


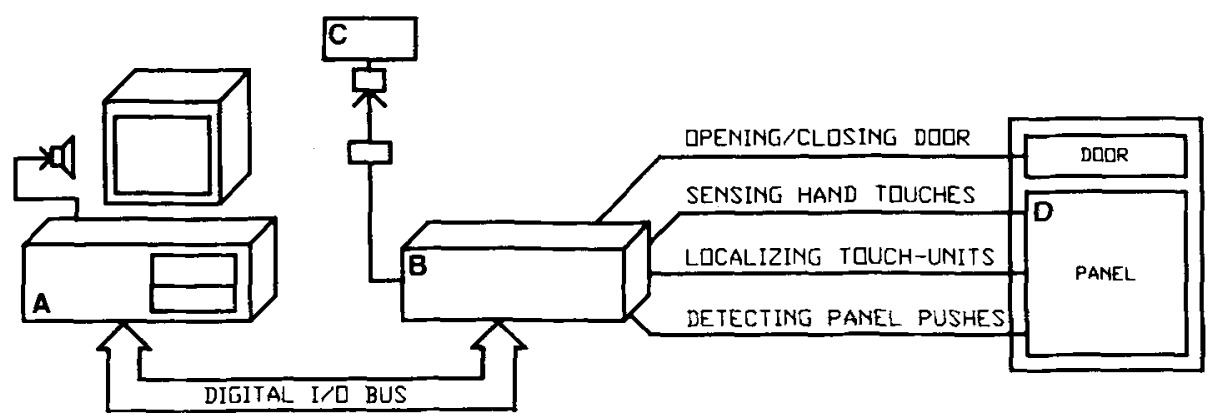

Figure 2. Box diagram of the main constituents of the system. (A) Computer. (B) Multifunction interface. (C) Food dispenser and its box relay. (D) Discriminanda.

the motor, or (2) through a contact relay (PRME 15005; C. P. Clare Inc., Wakefield, MA) within the interface. To detect palpation. of the stimuli (Figure 3C), any hand contact with a touch unit shifts the voltage of that unit (from $+5 \mathrm{~V}$ to $0 \mathrm{~V}$ ) and then modifies (from $0 \mathrm{~V}$ to $+5 \mathrm{~V}$ ) the output level of the 4584 inverter connected to the digital I/O bus. Finally, panel pushes are detected when one to four microswitches (B62-20Y; Bonnella Switches), attached to the back of each panel, are closed, changing (from $+5 \mathrm{~V}$ to $0 \mathrm{~V}$ ) the voltage of the output line (Figure 3D).

\section{TEST ADMINISTRATION PROGRAM}

The program, which is written in Turbo Pascal (Borland, Version 5.5) for MS-DOS computers, occupies 70K of memory. It consists of several executable files and a header file, where independent variables are set. The header can be edited by the user to customize the operation of the program. The test-administration program initially displays a menu with five choices: (1) Shaping phase 1 for monkey, (2) Shaping phase 2 for monkey, (3) monkey testing, (4) human testing, (5) exit.

\section{Shaping Phase 1 for Monkeys}

This subprogram is designed to train monkeys to insert their hands inside the box and touch the stimuli. It displays two $12 \times 12$ grids, representing the two rear panels, where the touch units are shown by shaded cells. The experimenter presses the " 1 ," " 2 ," or " 3 " key in order to open the left door, the right door, or both. Once this is done, a warning tone alerts the monkey that a trial will start and the door(s) is (are) opened. Any hand contact with the touch units (1) causes its corresponding cell on the computer's screen to turn black; (2) releases a Noyes pellet from the dispenser; and (3) ends the trial by closing the door(s). The time delay between the trial's auditory start signal and the opening of the door(s) as well as the maximum duration of door opening are coded in the header. No data are recorded during this phase of shaping.

\section{Shaping Phase 2 for Monkeys}

This subprogram is designed to train monkeys to discriminate the tactual stimuli and to push the panel. It differs in several ways from the previous one. First, this subprogram requires the experimenter to give the subject's name and the panel (left or right, corresponding to the " 1 " or " 2 " key) on which the "correct" stimulus is set. Second, in order to obtain a reward, the monkey has to touch the stimuli and then push the "correct" panel to activate the microswitches. A push on the "incorrect" panel is indicated by a tone and ends the trial. Third, the following data are automatically recorded by the computer: (1) latency; (2) the touch time for the left and right stimuli; (3) the global touch time; (4) accuracy. The signal to begin timing $\left(t_{0}\right)$ is given from the computer at the time the software triggers the door opening. The latency is thus defined as the time elapsed from $t_{0}$ to the first contact of the hand with a stimulus $\left(t_{1} ;\right.$ latency $\left.=t_{1}-t_{0}\right)$. The touch time for each stimulus corresponds to the total amount of time that each stimulus has been touched from $t_{1}$ to the push of the panel $\left(t_{2}\right)$. The global touch time corresponds to the time elapsed from $t_{1}$ to $t_{2}$ (global touch time $=t_{2}-t_{1}$ ). Accuracy refers to whether or not the animal pushes the "correct" panel. The time delay between the push of the panel and food or tone delivery and the delay between food or tone delivery and door(s) closing are defined in the header. All time measurements are in milliseconds.

\section{Monkey Testing}

This subprogram provides a more detailed output than that of the Shaping Phase 2 subprogram. Table 1 is a printout of the output of the monkey testing subprogram. The first two lines of the printout list the name of the subject, the date, and the hour of testing. The following data are then provided: (1) the number of the trial; (2) the door that was opened ( 1 for left; 2 for right; 3 for both); (3) the identification of the "correct" panel (1 for left; 2 for right); (4) the latency; (5) the touch time for the left stimulus; (6) the touch time for the right stimulus; (7) the global 

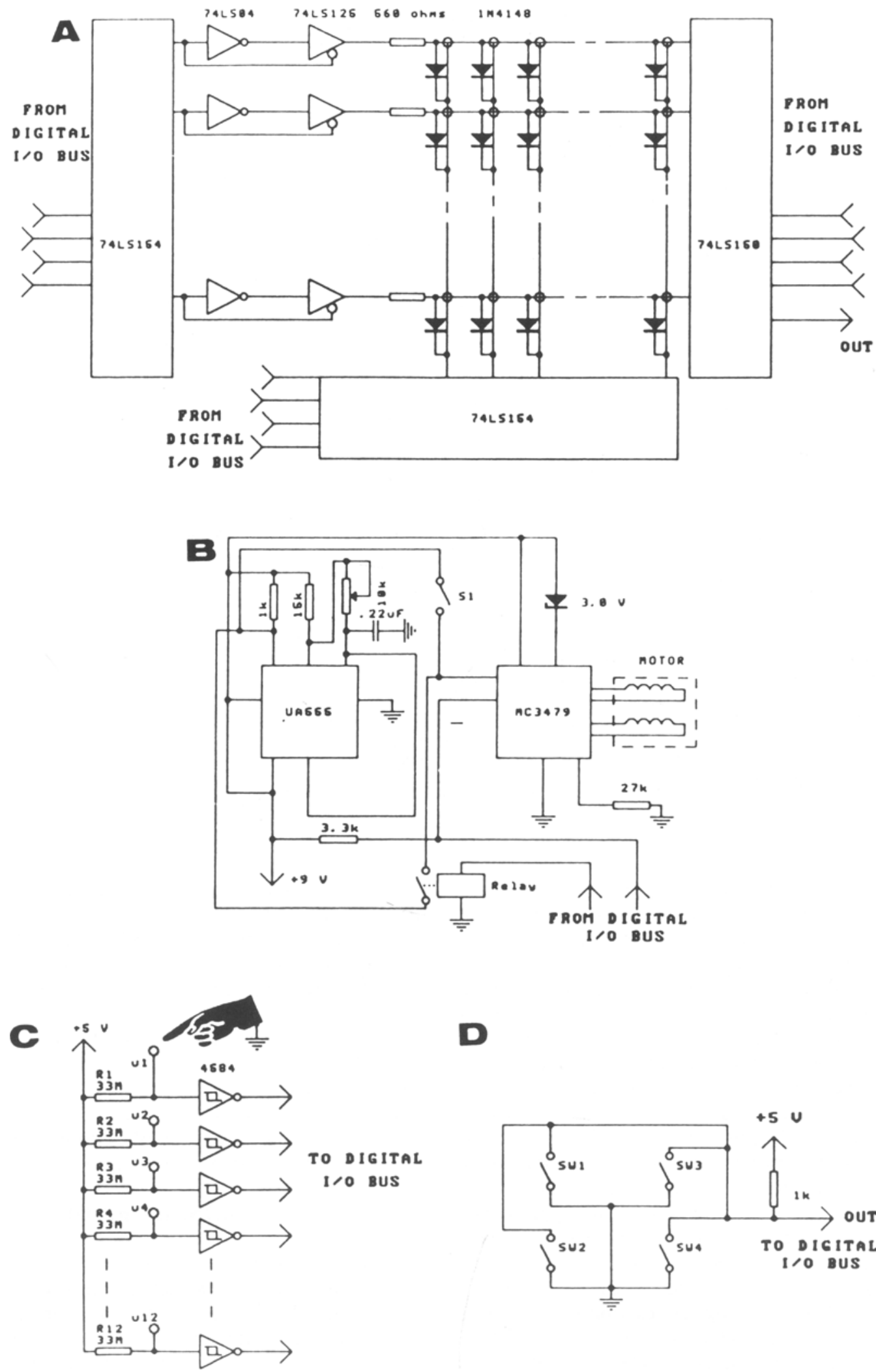

D

Figure 3. Circuit diagrams for the functions of (A) localization of the touch units on the rear panel, (B) front door opening and closing, (C) stimuli palpation detection, and (D) detection of panel pushes. 
Table 1

Printout of the Monkey Testing Subprogram

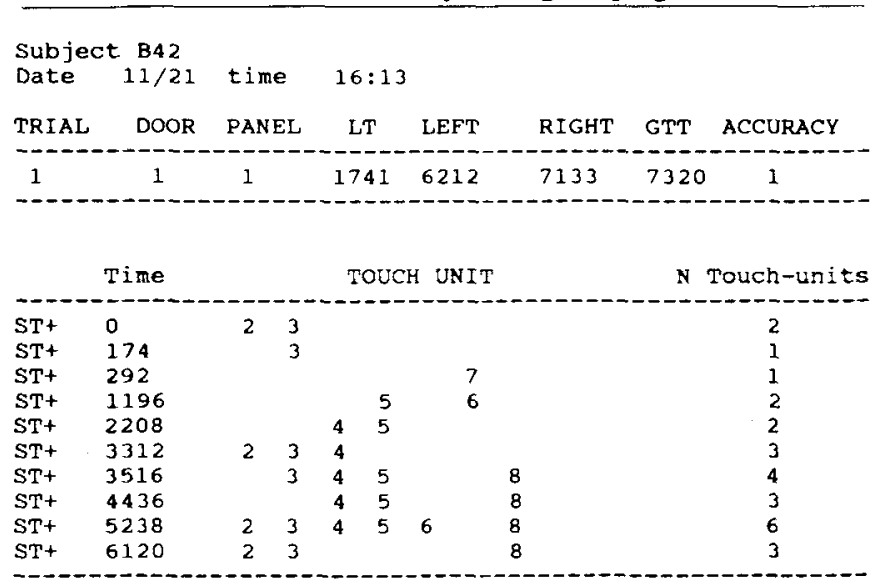

Number of states: 10

Maximum number of units simultaneously touched: 6

Mean number of units simultaneously touched: 2.8

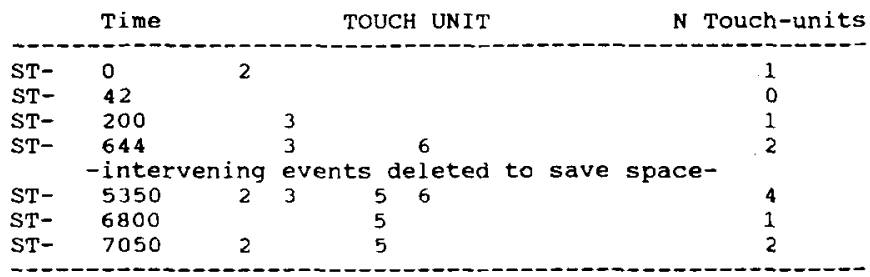

Number of states: 19

Maximum number of units simuitaneously touched: 4

Mean number of units simultaneously touched: 2.74

Note-LT = latency time. GTT = global touch time. Time is given in milli-

seconds.

touch time; and ( 8 ) the accuracy ( 1 for an accurate push; 0 for a mistake). These data are followed by data on the palpation of the "correct" (ST + ) stimulus. In this section, each line refers to a state of the manipulation. A state is defined as a pattern of units touched simultaneously. A state is entered when the subject touches a new pattern of units. Column 2 indicates the time in milliseconds when that state was entered, with the time " 0 " corresponding to the first hand contact with the "correct" stimulus (ST + ). The last column indicates the total number of units simultaneously touched in that state. Numbers in the intermediate columns correspond to the identification of the units that are palpated. In the example provided in Table 1, from Time 0 to 174 (in milliseconds), the subject touched Units 2 and 3 of ST+, whereas the subject touched the units numbered $2,3,4,5,6$, and 8 , corresponding to a sum of 6 units, from Time 5,238 to Time 6,120. This chronological description of the manner in which the subject explores the ST + allows the calculation of three indices of hand strategy. The first is the total number of states. The second is the largest number of units touched simultaneously during the palpation of $\mathrm{ST}+$. The third index is the average number of units touched during the investigation of ST + . The latter index corresponds to the sum of the number of units touched for all states in the palpation of ST +, divided by the number of states. In Table 1, the ST + section is followed by a printout of comparable data and measures for the "incorrect" stimulus (ST-). The program skips the STsection when only one stimulus is used.

\section{Human Testing}

This subprogram has the same characteristics as the previous one's except that pushes do not deliver a food pellet or produce an auditory signal.

\section{PRELIMINARY FINDINGS}

In a preliminary study (Fagot \& Vauclair, 1991), human subjects had to monohaptically investigate one stimulus composed of 8 touch units, and then recognize and point with one hand to an outline drawing of the touched stimulus that was visually presented along with two outlines of different stimuli. Results from 24 right-handed adults (12 males and 12 females) indicate no significant difference between the hands in terms of accuracy, but 
significant hand differences in how stimuli were palpated. On the average, the left hand simultaneously explored a greater number of touch units than the right hand did. The detailed quantitative measures available from this apparatus thus appear to offer interesting new ways to address the question of manual and hemispheric lateralization in haptic discrimination.

\section{CONCLUSIONS}

The apparatus described herein was initially designed to study hand lateralization in the discrimination of nonsense shapes. However, this instrument can also be used to study a variety of problems either in manual laterality or in object manipulation, recognition, or representation. Considering hand laterality, it is possible to use stimuli of high verbal codability, such as touch units arranged into letter shapes (e.g., H, I, P, L). Combined with other existing systems for delivering the stimuli in the visual mode (e.g., tachistoscopic presentation), this instrument is also adequate to address the question of the differences between intramodal (tactile-tactile) and intermodal (visual-tactile or tactile-visual) recognitions. Within other theoretical perspectives, the system may be a useful tool for investigating the problem of mental rotation, mirror image discrimination, or memory factors.

Program availability. A free copy of the source code and executable programs may be obtained by sending an MS-DOS formatted 5.25- or 3.5-in. disk to the first author. Please note that our interface system has to be duplicated in order for the program to run.

\section{REFERENCES}

Cohen, G. (1973). Hemispheric difference in serial versus parallel processing. Journal of Experimental Psychology, 97, 349-356.
Fagot, J., Dréa, C., \& Wallen, K. (1991). Asymmetrical hand use in the rhesus monkey (Macaca mulatta) in tactually and visually regulated tasks. Joumal of Comparative Psychology, 105, 260-268.

FAGOT, J., VAUCLAIR, J. (1991). Haptic discrimination of non-sense shapes: Exploratory strategies but not accuracy reveal laterality effects and sex differences. Manuscript submitted for publication.

Gardner, E. B., Englsh, A. G., Flannery, B. M., Hartnett, M. B. MacCormick, J. K., Wilhelmy, B. B. (1977). Shape-recognition accuracy and response latency in a bilateral tactile task. Neuropsychologia, 15, 607-616.

GroGAN, S. (1986). Hemispheric laterality for spatial analysis in children with reading problems. Perceptual \& Motor Skills, 63, 537-538.

Hoerster, W., EtTLinger, G. (1985). An association between hand preference and tactile discrimination performance in the rhesus monkey. Neuropsychologia, 23, 411-413.

LenharT, R. E., SCHWARTZ, S. M. (1983). Tactile perception and the right hemisphere: A masculine superiority for imagery coding. Brain \& Cognition, 2, 224-233.

NebEs, R. D. (1978). Direct examination of the cognitive function of the right and left hemisphere. In M. Kinsbourne (Ed.), Asymmetrical function of the brain (pp. 99-140). Cambridge: Cambridge University Press.

Summers, D. C., Lederman, S. J. (1990). Perceptual asymmetries in the somatosensory system: A dichhaptic experiment and critical review of the literature from 1929 to 1986. Cortex, 26, 201-226.

WITELSON, S. F. (1974). Hemispheric specialization for linguistic and non-linguistic tactual perception using a dichotomous stimulation technique. Cortex, 10, 3-17.

\section{NOTE}

1. With a small array of stimuli, units could be assembled side by side beforehand on a plastic baseboard (see Fagot \& Vauclair, 1991). At the start of each trial, the baseboard would be fixed on the rear panel of the box. This method eliminates useless manipulations during the experimental period and thus reduces the time needed to install each stimulus.
(Manuscript received January 29, 1991 ; revision accepted for publication July 17, 1991.) 